\title{
Research
}

Alison Cooper, Andrew Carson-Stevens, Michelle Edwards, Freya Davies, Liam J Donaldson, Pippa Anderson, Matthew Cooke, Jeremy Dale, Bridie Angela Evans, Barbara Harrington, Julie Hepburn, Peter Hibbert,

Thomas Hughes, Alison Porter, Aloysius Niroshan Siriwardena, Helen Snooks and Adrian Edwards

\section{Identifying safe care processes when GPs work in or alongside emergency departments:}

\author{
a realist evaluation
}

\begin{abstract}
Background

Increasing pressure on emergency services has led to the development of different models of care delivery including GPs working in or alongside emergency departments (EDs), but with a lack of evidence for patient safety outcomes.
\end{abstract}

\section{Aim}

This study aimed to explore how care processes work and how patient safety incidents associated with GPs working in ED settings may be mitigated.

\section{Design and setting}

Realist methodology with a purposive sample of 13 EDs in England and Wales with different GP service models. The study sought to understand the relationship between contexts, mechanisms, and outcomes to develop theories about how and why patient safety incidents may occur, and how safe care was perceived to be delivered.

\section{Method}

Qualitative data were collected lobservations, semi-structured audio-recorded staff interviews, and local patient safety incident reports). Data were coded using if, then, because' statements to refine initial theories developed from an earlier rapid realist literature review and analysis of a sample of national patient safety incident reports.

\section{Results}

The authors developed a programme theory to describe how safe patient care was perceived to be delivered in these service models, including: an experienced streaming nurse using local guidance and early warning scores; support for GPs' clinical decision making, with clear governance processes relevant to the intended role (traditional GP approach or emergency medicine approach): and strong clinical leadership to promote teamwork and improve communication between services.

\section{Conclusion}

The findings of this study can be used as a focus for more in-depth human factors investigations to optimise work conditions in this complex care delivery setting.

\section{Keywords}

clinical decision making; emergency

departments; general practitioners; human

factors; patient safety.

\section{INTRODUCTION}

Patient safety is described by the World HealthOrganizationasaseriousglobal public health concern, ${ }^{1}$ but new healthcare service models are frequently introduced without evidence for patient safety outcomes. An example is the implementation of GP services in or alongside EDs, advocated (and resourced) in England as an approach to manage increasing patient demand. ${ }^{3}$ As a result, these service models have increased in England from $81 \%$ to $95 \%$ (2017-2019), ${ }^{4}$ despite a lack of evidence for their effectiveness and safety outcomes. ${ }^{5}$

Urgent and emergency healthcare services are complex adaptive sociotechnical systems. ${ }^{6}$ The environment is unpredictable and challenging, with pressures of time and uncertainty, as a wide variety of patients present with undifferentiated problems. $^{7}$ GP service models associated with EDs may be situated inside the ED, integrated with the emergency medicine service linsideintegrated) or as a separate parallel service (inside-parallel); or outside the ED, on the

A Cooper, PhD, MRCGP, clinical research fellow; A Edwards, MBBS, PhD, professor of general practice; $\mathbf{M}$ Edwards, $\mathrm{PhD}$, research associate; A Carson-Stevens, PhD, MRCGP, clinical reader of patient safety and quality improvement; F Davies, PhD, MRCGP, clinical research fellow, Division of Population Medicine, School of Medicine, Cardiff University, Cardiff, UK. B Harrington, public contributor; J Hepburn, public contributor, PRIME Centre Wales.

BA Evans, PhD, research officer: A Porter $\mathrm{PhD}$, associate professor health services research; H Snooks, PhD, professor health services research, Institute of Life Science 2 Swansea University Medical School, Swansea, UK. LJ Donaldson, MD, FRCP, FFPH, chair of public health, London School of Hygiene and Tropical Medicine, London, UK. P Anderson $\mathrm{PhD}$, associate professor, Swansea Centre for Health Economics, Swansea University. M Cooke PhD, FRCS, FRCP, FRCEM, clinical professor J Dale, PhD, FRCGP, professor of primary care, Division of Health Sciences, Warwick Medical hospital site (outside-onsite) or separate from the hospital site (outside-offsite).' Previous analysis of a sample of national patient safety incident reports describing diagnostic error associated with these service models (UK Coroners' and National Reporting and Learning System reports) highlighted key areas for improvement, including: streaming processes; GPs' clinical decision making; and communication between services. ${ }^{8}$ Understanding how work conditions may influence the way GPs work (human factors) and how processes can be optimised to mitigate such events and support GPs in these different service models is overdue.

Quantitative analysis of routinely collected hospital data may not capture the complexity of these services, how they work, and why outcomes may occur, and may also be limited by poor data quality. Qualitative methods are required to improve understanding about how complex nonlinear phenomena may contribute to patient safety incidents (a 'Safety-l' approach). ${ }^{10}$ They can also be used to explore how

School, Coventry, UK. P Hibbert, PhD, program manager, Australian Institute of Health Innovation, Macquarie University, Australia. T Hughes, OBE, MSc, MBA, FRCS, MRCP, FRCEM, consultant in emergency medicine, John Radcliffe Hospital, Oxford, UK. AN Siriwardena, PhD, MMedSci, FRCGP, professor of primary and prehospital healthcare, School of Health and Social Care, University of Lincoln, Lincoln, UK.

\section{Address for correspondence}

Alison Cooper, 8th Floor Neuadd Meirionnydd, Division of Population Medicine, School of Medicine, Cardiff University, Cardiff CF14 4YS, UK

Email: CooperA8Acardiff.ac.uk

Submitted: 2 February 2021; Editor's response: 13 April 2021; final acceptance: 17 May 2021. (C)The Authors

This is the full-length article (published online 19 Oct 2021) of an abridged version published in print. Cite this version as: $\mathbf{B r} \mathbf{J}$ Gen Pract 2021; DOI: https://doi.org/10.3399/BJGP.2021.0090 


\section{How this fits in}

New healthcare service models are often introduced without evidence for patient safety outcomes; this includes GPs working in or alongside emergency departments (EDs). In the present study, realist methodology was used to explore what works, for whom, in what circumstances, and how, in a purposive sample of $13 \mathrm{EDs}$, to understand how and why patient safety incidents may occur, and how safe care was considered to be delivered. Working in ED settings may influence GPs' clinical decision making: working with a usual GP approach a more cautious GP approach; choosing for different patients whether to maintain a GP approach or adopt an emergency medicine approach; or adopting an emergency medicine approach. Experienced streaming nurses, clear governance processes to support the intended GP role, and strong clinical leadership to encourage communication and teamwork between services were perceived to facilitate safe patient care in these complex care delivery settings.

human factors enable work to be conducted safely in both expected and unexpected conditions, understanding work-as-done rather than work-as-intended la 'Safety-II' approach). ${ }^{11}$ Theory-driven realist methods are well suited to evaluating such services to explain what works, for whom, in what circumstances, and why, incorporating formal theory to describe how contextual factors may facilitate or inhibit patient safety outcomes. $^{12}$

This study aimed to test and refine initial theories developed through an earlier rapid realist review, ${ }^{13}$ and analysis of national

\section{Box 1. Realist definitions}

Context Pre-existing conditions that influence the success or failure of different interventions or programmes

\begin{tabular}{ll}
\hline Mechanism & $\begin{array}{l}\text { Characteristics of the intervention and people's reaction to it; how it influences their } \\
\text { reasoning }\end{array}$ \\
\hline Outcome & $\begin{array}{l}\text { Intended and unintended results of the intervention because of a mechanism } \\
\text { operating within a context }\end{array}$ \\
\hline Initial rough theory & $\begin{array}{l}\text { An early theory, informed by available evidence, about how, why, for whom, and in } \\
\text { what circumstances the intervention is thought to work, described as a context- } \\
\text { mechanism-outcome configuration }\end{array}$ \\
\hline Refined theory & $\begin{array}{l}\text { An initial theory that has been refined using primary or secondary evidence } \\
\text { Programme theory }\end{array}$ \\
$\begin{array}{ll}\text { An overall high-level theory summarising how the intervention works, developed } \\
\text { using the theories refined from the data }\end{array}$ \\
\hline Formal theory & $\begin{array}{l}\text { Existing social theories used as a lens through which to examine the data; otherwise } \\
\text { known as middle-range or substantive theory }\end{array}$ \\
\hline
\end{tabular}

patient safety incident reports, ${ }^{8}$ with qualitative data from a purposive sample of 13 case study sites, to explain how care processes are most likely to prevent or mitigate patient safety incidents associated with GPs working in ED settings.

\section{METHOD}

Realist methodology is a theory-driven approach to evaluation, identifying mechanisms that explain how or why contexts relate to outcomes to generate theories described as context-mechanismoutcome (CMO) configurations; specific terminology is defined in Box 1.12 This study followed RAMESES reporting and publication standards (see Supplementary Table S1). ${ }^{14}$

\section{Case site selection}

Case sites (hospitals) were recruited from responders to a national survey, followed up by a key informant telephone interview with the site clinical lead..$^{15}$ An online survey was sent to the clinical directors of all type 1 EDs (24-hour consultant-led units with full resuscitation facilities) in England ( $n=171)$ and Wales $(n=13)$ on behalf of both the 'GPs in EDs'"16 and 'General Practitioners and Emergency Departments' (GPED) ${ }^{17}$ study teams (both with the same funder). The aim was to capture data about the GP services being provided in or alongside EDs and how they worked, to inform a taxonomy of GP-ED models for both studies. The published taxonomy contains further information about the survey process and results.?

This study had survey responses from 71 English and 6 Welsh sites $(n=77 / 184$ $42 \%)$. The GPED team also provided data for 41 English departments from Care Quality Commission reports and NHS England, totalling information on $62 \%(n=118 / 189)$ of type 1 EDs in England and Wales. ${ }^{7}$ As a gauge of non-response bias, the study's 71 English survey responders included $82 \%$ ( $n=58 / 71)$ who had applied for capital bid (GP streaming) funding, compared with $84 \%$ of the 100 non-responders in England. The 13 case sites were purposively selected according to variables listed in Box 2 to ensure they covered a range of models and contexts. The included sample of anonymised case study sites and characteristics is listed in Supplementary Table S2. Classified by the taxonomy, these included:

- three 'inside-integrated' models;

- four 'inside-parallel' models lone was reclassified following the visit); 


\section{Box 1. Variables used to purposively sample emergency departments}

- GP service implemented in the emergency department since 2010

- Different service models: inside-integrated; inside-parallel; outside-onsite; and sites with no GP service

- Spread of geographical locations in England and Wales

- Variety of contexts - including hospitals in rural and urban locations/towns, small and large hospitals, and higher versus lower attendances

- Variation in patient streaming methods - who streams, streaming criteria, and guidance

- Variation in the physical layout of the GP service in relation to the ED

- Variation in relationships with the GP out-of-hours service contexts may result in intended and unintended outcomes; see Supplementary Table S3 for an example of the interview guide. $^{18}$

Local patient safety incident reports. Up to four separate requests were made for reports relevant to the GP service at each participating site lexcluding those with no GPs). These data were usually in the form of printed anonymised reports that were given, in person, to the researcher who copied the free text directly onto a remotely accessed secure computer platform (PISA platform) at Cardiff University.

- three 'outside-onsite' models; and

- three sites without a GP service model.

\section{Data collection}

Two researchers visited all sites with a GP service $(n=10)$ for $2-4$ days (mean 3 days) and individually conducted a 1-day visit at control sites between January 2018 and April 2019. The study conducted observations, including informal interviews: semi-structured audio-recorded realist interviews; and analysed local patient safety incident reports.

Observations. The authors spent time in reception and clinical areas (but did not observe clinical consultations) and observed triage and streaming processes. The authors opportunistically introduced themselves to a wide range of staff and asked questions to test various theories. When it was not possible to talk with staff, the authors observed how the systems worked, taking handwritten fieldnotes that were typed the same evening.

The authors met every 2 hours during the day to discuss findings, refer to the list of initial theories, and identify evidence gaps for theory testing. Eight visits were conducted midweek lusually Monday to Wednesdayl, with six visits, including observations, in the evening. Two visits were conducted over a weekend. Where possible, an exit interview was held with the clinical director, before leaving, to assist theory refinement.

Staff realist interviews. The clinical director, ED staff, and GPs were recruited during case site visits for audio-recorded interviews on site in a private area, or, later, via telephone; these were then transcribed verbatim. The realist teacher-learner interview technique was used where initial theories are presented to the participant to explore how mechanisms in different

\section{Data analysis}

Themes were analysed based on the initial theories generated through a rapid realist review, ${ }^{13}$ and analysis of national patient safety incident reports (Table 1). ${ }^{8}$ NVivo (version 11) was used to support categorisation of data, with separate folders for documents relevant to each GP service model linside-integrated, insideparallel, outside-onsite, and no GPs). The authors coded data using 'if, then, because' statements to capture the nuance of different contexts. ${ }^{19}$ The level of qualitative evidence was classified supporting these ethnography principles. ${ }^{20}$ Findings were discussed weekly within the study team and co-applicants, including patient and public representatives, going back to the data for further information or clarification as required.

\section{Data synthesis}

High-level themes and positive and negative outcomes, grouped with mechanisms at individual, department, and wider system levels, were used as a coding framework to categorise the statements across folders. The authors then used Microsoft Excel to consolidate statements into $\mathrm{CMO}$ configurations. ${ }^{19}$ The authors mapped CMO configurations developed for each GP service model between service models, synthesising using Pawson's theory-building processes (juxtaposition, reconciliation, adjudication, and consolidation). ${ }^{21}$ The authors then developed a master Excel file to capture the whole process and populate the evidence (where available) for refined CMO configuration development.

\section{Incorporating formal theory}

The authors then incorporated Croskerry's dual-process model of reasoning to help explain GPs clinical decision making in ED settings. ${ }^{12,22}$ The model is based on statements in a hierarchy based on meta- 


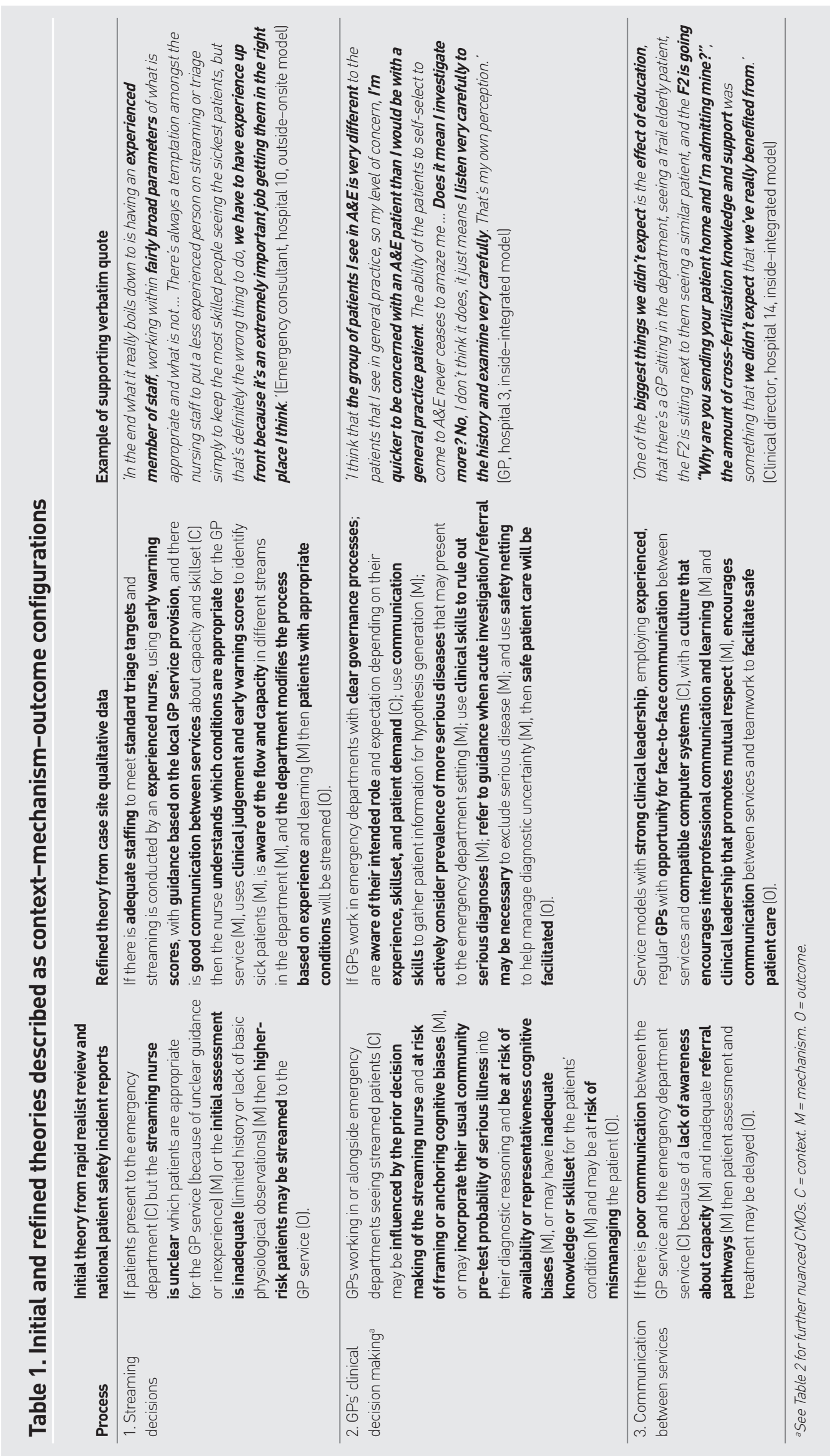




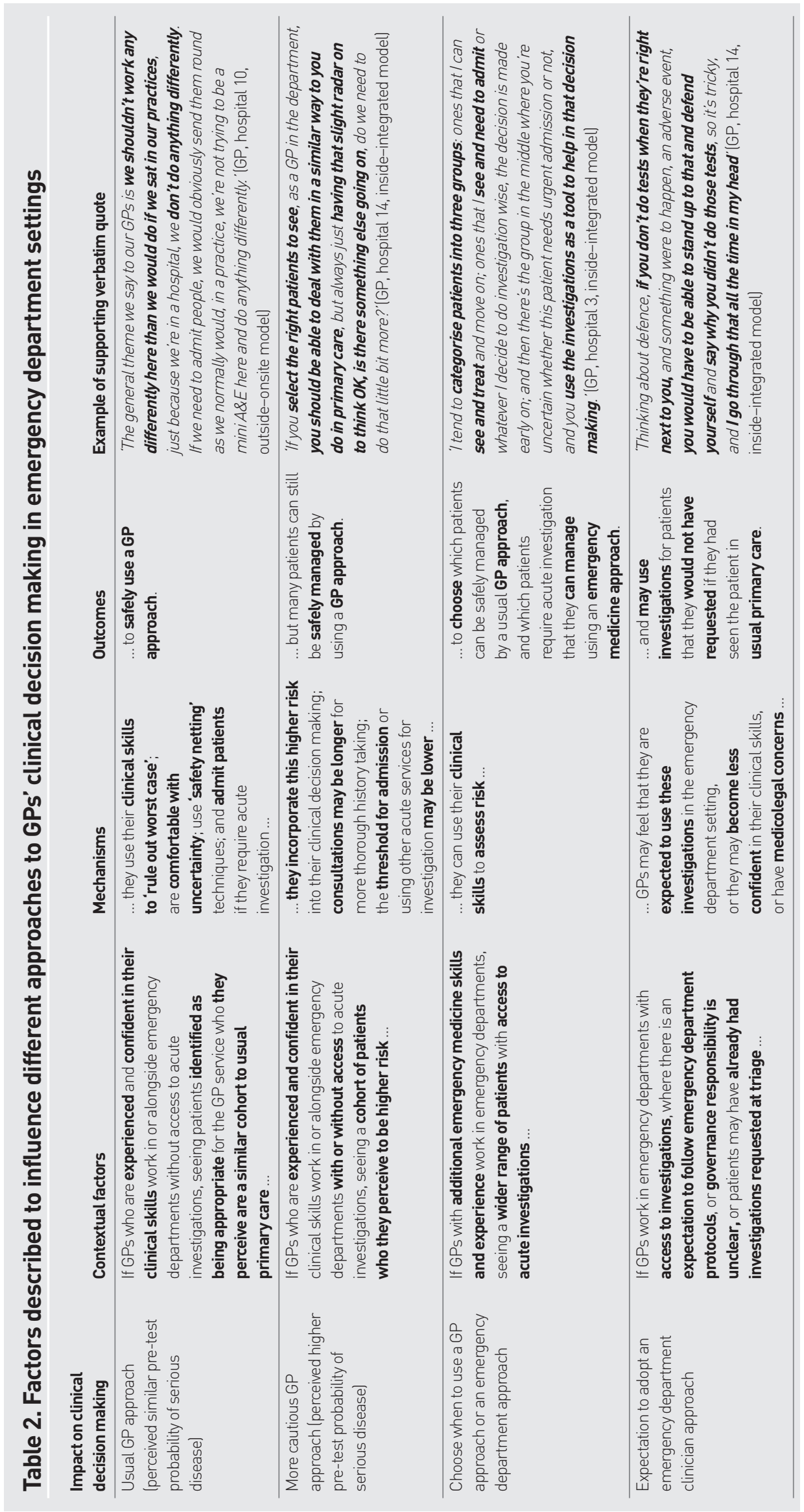


two distinct decision-making processes: 'System I' and 'System II', originally described by Kahneman. ${ }^{23}$ 'System I' is fast, effortless, intuitive, and automatic; it is typical in diagnostic decision making by experienced clinicians who rely on pattern recognition or shortcuts (heuristics). 'System II' is slow, laborious, and logical.23 Croskerry applied this to clinical medicine and specifically to ED settings, describing the risks of cognitive biases in these settings. ${ }^{22,24-26}$ Findings were structured around the diagnostic process of generation, evaluation, and verification. ${ }^{25,26}$

\section{Stakeholder feedback}

A national stakeholder event was held in Bristol, in December 2019, with a wide range of English- and Welsh-based attendees $(n=56)$, including policymakers and commissioners $(n=4)$, managers $(n=6)$, patient and public contributors ( $n=13)$, ED doctors $(n=6)$, nurse practitioners $(n=2)$, GPs $(n=5)$, academics including study co-applicants ( $n=17)$, and administrators $(n=3)$. Results were presented and feedback was collected from small-groupfacilitated discussions.

\section{Patient and public involvement}

Patients and public members were involved in the study design and co-applicants in the funded study. ${ }^{16}$ They used their experience as NHS patients to contribute to this research. They supported recruitment and involvement of public and patient contributors to the stakeholder event. They were involved in discussing the draft data and preparing this article. ${ }^{27}$

\section{RESULTS}

The authors included data from 66 staff interviews (Supplementary Table S4), fieldnotes from researcher observations at the purposive sample of 13 case sites, and 14 local patient safety incident reports relevant to the GP services (see Supplementary Table S5).

Clinical directors from nine of the 10 hospitals with a GP service had no patient safety concerns and did not describe any patient safety experiences related to the GP service. Two clinical directors from insideintegrated model sites perceived that, since GPs had been working in the department, overall patient safety had improved because more experienced, permanent GPs could also give advice to other staff members (hospitals 3 and 8). Safety incidents (and potential risks) regarding the GP service were described by senior staff at one case site with a GP service linside-parallel model) and at a site that no longer had GPs working there. These supported the authors' initial theories developed through earlier analysis of a sample of national patient safety incident reports describing diagnostic error associated with GP service models. $^{8}$

Refined theories from these case site qualitative data focused on staff perceptions about how patient safety incidents, described in the authors initial theories, could be mitigated. They are presented under the following care processes: facilitating appropriate streaming decisions; supporting GPs' clinical decision making; and improving communication between services (Table 1).

\section{Facilitating appropriate streaming decisions}

Streaming nurses having difficultyidentifying patients with appropriate conditions for the GP service was a common theme reported by ED doctors, nurses, and GPs across many case study sites (hospitals 4, 6, 9, and 10):

'It's a bit hit and miss, it depends on what the help of the triage nurse is ... sometimes patients you're seeing are inappropriate, I've seen epiglottitis, which, really, I shouldn't be seeing as a GP in A\&E, but there's lots of things that I could be seeing, which I don't end up seeing, because they're deemed to be an A\&E case.' (GP, hospital 4, insideparallel model)

An experienced advanced nurse practitioner described junior triage nurses inexperience as negatively influencing streaming decision making (hospital 10). He described how inexperienced nurses may not explore why patients had presented to the ED with the risk of missing red flag' symptoms, such as the possibility of cauda equina syndrome when a patient with chronic back pain presents with a history of incontinence. Understaffing in one case study site was also reported to delay the streaming process and triage because the streaming nurse also had to administer treatments. Many hospital case study sites were happy to share learning about how and why the streaming process worked well and how it had been modified, such as measuring basic observations, to ensure appropriate patients were streamed to the GPs:

'So, to give you an example of how we've learned ... we had a child seen in the triage room, had the eyeball, went to Urgent Care 
... thankfully, the GP picked up that this was a sick child, got them to the resus room, ended up in intensive care. So, we had a very rapid learning and a very rapid PDSA [Plan Do Study Act] cycle there. ' (Clinical director, hospital 3, inside-integrated model)

Guidance relevant to the local GP service was considered important but an experienced streaming nurse who could use their clinical judgement was felt to be essential (hospitals 7, 9, and 10). Appropriate communication between services allowed the streaming nurse to understand the capacity of the different streams (situation awareness), which again influenced streaming decisions (hospital 10).

\section{Supporting GPs' clinical decision making}

There was some evidence of GPs working within integrated service models seeing a wider range of patients, with some reports of fracture mismanagement (hospitals 4 and 14) and not following standard ED child safeguarding protocols (hospitals 3 and 4):

A child was seen who was known to have had input from social services ... and the GP had seen them, and they really should have rung social services just to alert them that the patient had been seen ... but they just seemed to maybe not have quite the right level of concern and appreciation of the need to keep social services involved. (Clinical director, hospital 4, inside-parallel model)

Unclear governance processes across different commissioning organisations, including job description, induction, and supervision requirements, were felt, by a senior consultant at one site, to contribute to confusion about which patients the GP service should be managing:

I was concerned from the outset, really, about the lack of clarity behind where was the governance, what were they supposed to be seeing, was it within their normal scope of practice... We had an incident of a missed cervical spine fracture. 'Emergency consultant, hospital 4, inside-parallel modell

Four $\mathrm{CMO}$ configurations were developed from GP interview data (Table 2) to describe how working in ED settings influenced (or did not influence) their use of acute investigations and clinical decision making: a usual GP approach; a more cautious GP approach; the choice to take a GP approach or an emergency medicine approach; and the expectation to adopt an emergency medicine approach. Croskerry's framework was then applied to consider the risks of cognitive errors at different stages of the diagnostic process and further refine these theories. ${ }^{25,26}$

Diagnosis generation. Generation of one or more diagnostic hypotheses begins early in the process, even before the clinical encounter with the patient has begun. ${ }^{25}$ GPs described making early clinical decisions, before the patient had been seen, based on the written triage notes, sending inappropriate patients back to the ED if necessary. Establishing the acuity of the patient's condition was a common strategy described by GPs working in ED settings: categorising patients into those who required immediate medical attention or investigation and those who did not, rather than focusing on a specific diagnosis:

'It's a different approach to working in the community where there's usually nothing serious - it's important not to miss a serious diagnosis. My approach: are there red flags? If not, can / treat it? Can I redirect?' (Comments from GP fieldnotes, hospital 9, inside-parallel model)

GPs' perceptions of the 'pre-test' prevalence of serious disease, and whether the cohort of patients was similar to usual primary care patients or a higher-risk group, was described to impact their clinical decision making. GPs who perceived the cohort of patients as higher risk described a different level of concern and management of risk in the ED than in usual primary care. Initial information gathering from the patient, to understand why they had presented to the ED that day, and the background of the presenting complaint, was described by some experienced GPs as key to diagnostic decision making.

Diagnostic evaluation. Many GPs described excluding serious disease by ruling out the 'worst case' as the priority, ${ }^{28}$ often through careful history and examination, even if acute investigations were available. However, some GPs described a lower threshold to admit patients for investigation to exclude serious disease than they would in the community setting because of the increased prevalence of serious illness in ED settings.

Diagnostic verification. GPs described the priority being to exclude serious disease rather than making an actual diagnosis, 
which may not be possible because of limitations of the service:

For me, my sort of mental triage system is "Do I need to admit you, yes or no, and can I deal with your issue now", i.e. is it long term, in which case I probably can't do very much, because I don't have access to all of your notes and it's not very practical, I can't organise blood tests, I can't organise scans ... in which case l'll have to send you back to your GP.' (GP, hospital 7, inside-parallel modell

The strategy of 'safety netting' was described as good practice to help manage diagnostic uncertainty - advising patients of potential worsening symptoms and when further medical advice should be sought. ${ }^{29}$

\section{Improving communication between services}

Some hospital case sites were observed or reported to have limited communication between the GP and ED services. Incompatible computer systems were linked to two patient safety incidents, where patient assessment and treatment had been delayed (hospitals 3 and 6) (Supplementary Table S5). Receptionists at another case site described how they had three different computer systems to operate (for the ED, the GP-ED service, and the GP out-of-hours service), which led to duplicate patient entries on different systems and increased the likelihood of patients becoming lost in or between the different systems (hospital 7).

The layout of the department, with distance between the services limiting face-to-face communication, was felt to contribute to very limited communication between services at one site (hospital 11):

We're not very integrated with the ED and we don't, we don't feel very integrated, it still feels a bit us and them.' (GP, hospital 11. outside-onsite model)

An 'us and them' culture was observed and reported at another site (despite there being good opportunity for face-to-face communication with the GPs working out of an ED cubicle). At this site, juniors were not encouraged to ask the GPs for advice (hospital 4).

Another site, however, with a separate GP service, reported good communication through the senior nursing team, reviewing on-the-day capacity and skillsets, and moving staff between services to meet patient demand. The integrated GP services reported good communication, which was perceived to promote interprofessional learning. At these sites the GPs were employed on a regular, rather than locum, basis and there were good opportunities for face-to-face communication. GPs were described, not only to give clinical advice, but also to provide advice on primary care referral pathways, which ED staff reported as helpful. The authors observed a sense of multidisciplinary respect, trust, and teamwork, with clear ED clinical leadership (hospitals 3, 8, and 14). Strong GP leadership was seen at several case study sites, and was also reported to improve communication between the services and perceived to improve patient safety (hospitals 3, 10, and 14).

\section{Programme theory}

This study's findings were summarised in a programme theory, conceptualising the complexity of patients and pathways, to describe factors perceived to facilitate GPs delivering safe patient care in or alongside EDs (Supplementary Figure S1).

\section{DISCUSSION}

\section{Summary}

A programme theory was developed from observations, incident reports, and in-depth realist interviews to describe how safe patient care was perceived to be delivered when GPs work in or alongside EDs: experienced streaming nurses using early warning scores and local guidance to facilitate appropriate streaming decisions; clear governance processes to support GPs' clinical decision making depending on the intended role (traditional GP or emergency medicine clinician); and compatible computer systems, experienced regular GPs, and strong clinical leadership to encourage communication and teamwork between the emergency and GP services.

\section{Strengths and limitations}

Thirteen case study sites were purposively recruited for theory testing and refinement lincluding different service models in different sized hospitals, geographically spread across England and Wales). These were visited by the same two researchers who applied a consistent realist approach, testing and refining initial theories developed from the literature, ${ }^{13}$ and analysis of national patient safety incident reports, ${ }^{8}$ through realist teacher-learner interview techniques to explore how human factors influenced clinical risk and work-as-done rather than work-as-intended, when GPs worked in these settings. ${ }^{11,18}$ Longer visits 
and observations of clinical consultations, rather than interview data subject to staff and researcher perceptions, would have provided stronger evidence about workas-done'. Quantitative data are required to understand the effects of GPs working in EDs on safety outcomes, and on comparative effects with other professional groups.

The work was conducted as part of a larger study that dictated the sampling approach. Selecting sites from a national survey, with a response rate of $42 \%$, limited sampling, although the authors had information on an additional 20\% of hospitals, collectively with no evidence of non-response bias. ${ }^{7}$ No sites were recruited where GPs screened patients at the front door in a gatekeeper role; however, there may be departments operating this service model, of which the authors were unaware.

\section{Comparison with existing literature}

GPs are recognised as low patient safety incident reporters, which may have contributed to the low number of local reports identified. ${ }^{30}$ There is little national guidance on which ED patients should be streamed to GP services, ${ }^{5,31}$ or by whom, with this study's work supporting an experienced senior nurse over algorithmic methods. $^{32}$

The dual-process model of reasoning has previously been applied to GPs working in a similar high-risk setting, out-of-hours, where they would not know their patients. ${ }^{33}$ Similar management approaches were described: dividing patients into those with serious (or potentially serious) conditions and patients likely to have non-serious conditions; and using 'safety netting' to manage diagnostic uncertainty. ${ }^{33}$ An initial patient-guided search, or the 'golden minute', is described as key in the information-gathering stage of the well-known Calgary-Cambridge clinical consultation. ${ }^{34,35}$ GPs described how they used their communication skills to gather information and to exclude serious disease, which may explain their reduced use of acute investigations. ${ }^{36}$

Communication failures, exacerbated by hierarchical differences and conflicting roles and role ambiguity, are associated with increased patient safety incidents, ${ }^{37,38}$ while interventions to improve communication between healthcare professionals, such as briefings or 'huddles', are associated with improved patient safety outcomes. ${ }^{39,40}$ Clinician involvement in leadership positions in hospitals is associated with improved quality of patient care..$^{41}$

\section{Implications for research and practice}

Since this work was conducted, urgent and emergency care services along with almost all NHS service provision have changed because of the COVID-19 pandemic, including telephone screening of ED 'walk-in' attendances ${ }^{42}$ and remote GP consultations. ${ }^{43}$ The learning from this work and human factors concepts can be applied when evaluating these new services for quality improvement purposes, including how streaming lor telephone screening and 'care navigation') decisions are made; how remote consultations may impact on GPs' clinical decision making; and how to promote communication between new emergency service models to ensure improved patient safety. ${ }^{44}$

The complexity of the ED setting and the patients presenting to it, who are often seen by more than one staff member who do not know them, or their previous state of health, provides challenges for staff, including GPs. The authors propose a programme theory to describe how safe care is perceived to be facilitated when GPs work in or alongside EDs, including: appropriate streaming decisions; supporting GPs' clinical decision making; and improving communication between services. These findings can be used as a focus for more in-depth human factors investigations to optimise work conditions in this complex care delivery setting.

\section{Acknowledgements}

The authors would like to thank study team members (Delyth Price, Nigel Pearson, Charlotte Bonner-Evans, Alan Watkins, and Saiful Islam) and staff at case study sites for their contributions to this work.

\section{Open access}

This article is Open Access: CC BY 4.0 licence (http://creativecommons.org/ licences/by/4.0/).

\section{Discuss this article}

Contribute and read comments about this article: bjgp.org/letters 


\section{REFERENCES}

1. World Health Organization. Patient safety. 2021. http://www.euro.who.int/en/ health-topics/Health-systems/patient-safety laccessed 22 Sep 2021).

2. McDonnell A, Wilson R, Goodacre S. Evaluating and implementing new services. BMJ 2006; 332(7533): 109-112.

3. NHS England. Transforming urgent and emergency care services in England. 2015. http://www.nhs.uk/NHSEngland/keogh-review/Documents/safer-fasterbetter-v28.pdf (accessed 22 Sep 2021).

4. Brant $\mathrm{H}$, Voss $\mathrm{S}$, Morton $\mathrm{K}$, et al. Current provision of general practitioner services in or alongside emergency departments in England. Emerg Med J $2021 ; \mathbf{3 8 ( 1 0 ) : ~ 7 8 0 - 7 8 3 . ~}$

5. Cooper A, Carson-Stevens A, Hughes T, Edwards E. Is streaming patients in emergency departments to primary care services effective and safe? BMJ 2020; 368: $\mathrm{m} 462$

6. Holland JH. Adaption in natural and artificial systems: an introductory analysis with applications to biology, control, and artificial intelligence. Cambridge, MA: MIT Press, 1992

7. Cooper A, Edwards M, Brandling J, et al. Taxonomy of the form and function of primary care services in or alongside emergency departments: concepts paper. Emerg Med J 2019; 36(10): 625-630.

8. Cooper A, Carson-Stevens A, Cooke M, et al. Learning from diagnostic error to enhance safety when GPs work in or alongside emergency departments: a mixed-method analysis of coroners' and patient safety incident reports. BMC Em Med 2021; under review.

9. Baracaia S, McNulty D, Baldwin S, et al. Mental health in hospital emergency departments: cross-sectional analysis of attendances in England 2013/2014. Emerg Med J 2020; 37(12): 744-751.

10. Bion J, Richardson A, Hibbert P, et al. 'Matching Michigan': a 2-year stepped interventional programme to minimise central venous catheter-blood stream infections in intensive care units in England. BMJ Qual Saf 2013; 22(2): 110-123.

11. Hollnagel E. Safety-I and Safety-II: the past and future of safety management. Farnham: Ashgate Publishing, 2014

12. Pawson R, Tilley N. Realistic evaluation. London: SAGE Publications, 1997.

13. Cooper A, Davies F, Edwards M, et al. The impact of general practitioners working in or alongside emergency departments: a rapid realist review. BMJ Open 2019; 9(4): e024501

14. Wong G, Westhorp G, Manzano A, et al. RAMESES II reporting standards for realist evaluations. BMC Med 2016; 14(1): 96 .

15. Edwards M, Cooper A Davies F et al. Emergency department clinical leads experiences of implementing primary care services where GPs work in or alongside emergency departments in the UK: a qualitative study. BMC Emerg Med 2020; 20(1): 62

16. Edwards A. Evaluating effectiveness, safety, patient experience and system implications of different models of using GPs in or alongside emergency departments. 2017. https://www.journalslibrary.nihr.ac.uk/programmes/ hsdr/1514504/\# laccessed 22 Sep 2021).

17. Morton K, Voss S, Adamson J, et al. General practitioners and emergency departments (GPED) - efficient models of care: a mixed-methods study protocol. BMJ Open 2018; 8(10): e024012

18. Manzano A. The craft of interviewing in realist evaluation. Evaluation 2016: 22(3): 342-360.

19. Pearson M, Brand SL, Quinn C, et al. Using realist review to inform intervention development: methodological illustration and conceptual platform for collaborative care in offender mental health. Implement Sci 2015; 10: 134

20. Noblit GW, Dwight Hare R. Meta-ethnography: synthesizing qualitative studies. Newbury Park, CA: Sage Publications, 1988

21. Pawson R. Realist synthesis: new protocols for systematic review. In: Evidencebased policy. London: Sage Publications, 2006; 73-104
22. Croskerry P. A universal model of diagnostic reasoning. Acad Med 2009; 84(8): 1022-1028.

23. Kahneman D. Thinking, fast and slow. London: Penguin, 2012

24. Croskerry P. Clinical cognition and diagnostic error: applications of a dual process model of reasoning. Adv Health Sci Educ Theory Pract 2009.

14(Suppl 1): 27-35.

25. Kovacs G, Croskerry P. Clinical decision making: an emergency medicine perspective. Acad Emerg Med 1999; 6(9): 947-952.

26. Kassirer JP, Kopelman RI. Cognitive errors in diagnosis: instantiation, classification, and consequences. Am J Med 1989; 86(4): 433-441.

27. Staniszewska S, Brett J, Simera I, et al. GRIPP2 reporting checklists: tools to improve reporting of patient and public involvement in research. BMJ 2017; 358: 33453.

28. Croskerry P. Achieving quality in clinical decision making: cognitive strategies and detection of bias. Acad Emerg Med 2002; 9(11): 1184-1204.

29. Neighbour R. Safety netting: now doctors need it too. Br J Gen Pract 2018; DOI: https://doi.org/10.3399/bjgp18X695849

30. Carson-Stevens A, Hibbert P, Williams H, et al. Characterising the nature of primary care patient safety incident reports in the England and Wales Nationa Reporting and Learning System: a mixed-methods agenda-setting study for general practice. Health Services and Delivery Research 2016; 4(27): 1-76.

31. Royal College of Emergency Medicine. Initial assessment of emergency department patients. 2017. https://www.rcem.ac.uk/docs/SDDC\%20Intial\%20 Assessment\%20(Feb\%202017).pdf (accessed 22 Sep 2021).

32. Iversen AKS, Kristensen M, Østervig RM, et al. A simple clinical assessment is superior to systematic triage in prediction of mortality in the emergency department. Emerg Med J 2019; 36(2): 66-71.

33. Balla J, Heneghan C, Thompson M, Balla M. Clinical decision making in a highrisk primary care environment: a qualitative study in the UK. BMJ Open 2012; 2(1): e000414

34. Kurtz S, Silverman J, Benson J, Draper J. Marrying content and process in clinical method teaching: enhancing the Calgary-Cambridge guides. Acad Med 2003; 78(8): 802-809.

35. Beckman HB, Frankel RM. The effect of physician behavior on the collection of data. Ann Intern Med 1984; 101(5): 692-696.

36. Medical Defence Union. Consultant quide. https://wnw.themdu.com/-/media/ files/mdu/publications/guides/mdu-consultant-pack.pdf laccessed 29 Sep 2021).

37. Sutcliffe KM, Lewton E, Rosenthal MM. Communication failures: an insidious contributor to medical mishaps. Acad Med 2004; 79(2): 186-194.

38. Leonard M, Graham S, Bonacum D. The human factor: the critical importance of effective teamwork and communication in providing safe care. Qual Saf Health Care 2004; 13(Suppl 1): i85-i90.

39. Makary MA, Mukherjee A, Sexton JB, et al. Operating room briefings and wrong-site surgery. J Am Coll Surg 2007; 204(2): 236-243.

40. Edelson DP, Litzinger B, Arora V, et al. Improving in-hospital cardiac arrest process and outcomes with performance debriefing. Arch Intern Med 2008, 168(10): 1063-1069

41. Sarto F, Veronesi G. Clinical leadership and hospital performance: assessing the evidence base. BMC Health Serv Res 2016; 16(Suppl 2): 169.

42. Discombe M. NHSE launches pilots to shake-up A\&E model. HSJ 2020; 30 Jun https://www.hsj.co.uk/coronavirus/nhse-launches-pilots-to-shake-up-aandemodel/7027943.article (accessed 22 Sep 2021).

43. Greenhalgh T, Koh GCH, Car J. Covid-19: a remote assessment in primary care BMJ 2020; 368: m1182.

44. Chartered Institute of Ergonomics \& Human Factors. Human factors and healthcare: evidencing the impact of human factors training to support improvements in patient safety and to contribute to cultural change. 2019 https://www.hee.nhs.uk/sites/default/files/documents/Health\%20Education\%20 England\%20and\%20CIEHF\%20-\%20Human\%20Factors\%20and\%20 Healthcare\%20Report.pdf (accessed 22 Sep 2021). 